\title{
Phenotypic Variability from Benign Infantile Epilepsy to Ohtahara Syndrome Associated with a Novel Mutation in SCN2A
}

\author{
Steffen Syrbe ${ }^{a, b} \quad$ Boris S. Zhorov ${ }^{f, g} \quad$ Astrid Bertsche $^{b} \quad$ Matthias K. Bernhard $^{b}$ \\ Frauke Hornemann ${ }^{b}$ Ulrike Mütze ${ }^{a, b}$ Jessica Hoffmann ${ }^{e}$ \\ Konstanze Hörtnagel ${ }^{\mathrm{e}}$ Wieland Kiess $^{\mathrm{b}}$ Franz W. Hirsch ${ }^{\mathrm{d}}$ Johannes R. Lemke ${ }^{c}$ \\ Andreas Merkenschlager ${ }^{\mathrm{b}}$ \\ a Division of Child Neurology and Inherited Metabolic Diseases, Department of General Pediatrics, Center for \\ Pediatrics and Adolescent Medicine, University Hospital Heidelberg, Heidelberg, ${ }^{\mathrm{b}}$ Department of Women and \\ Child Health, Hospital for Children and Adolescents, and 'Institute of Human Genetics, and d Department of Imaging \\ and Radiotherapy, Section Pediatric Radiology, University of Leipzig Hospitals and Clinics, Leipzig, and ${ }^{~} \mathrm{CeGaT}^{\mathrm{C}}$ \\ $\mathrm{GmbH}$, Tübingen, Germany; ${ }^{\mathrm{f} D e p a r t m e n t}$ of Biochemistry and Biomedical Sciences, McMaster University, Hamilton, \\ Ont., Canada; ${ }^{9}$ Sechenov Institute of Evolutionary Physiology and Biochemistry, Russian Academy of Sciences, \\ St. Petersburg, Russia
}

\section{Key Words}

Brain atrophy · Channelopathy · Early-onset epileptic encephalopathy · Epilepsy

\begin{abstract}
Mutations in SCN2A have been associated with benign familial neonatal-infantile seizures (BFNIS) as well as infantile-onset epileptic encephalopathy, such as Ohtahara syndrome (OS). We describe a family with 3 affected individuals carrying the novel SCN2A missense variant c.1147C>G, p.Q383E affecting a residue proximal to the highly conserved selectivity filter in the P-loop of the voltage-gated sodium channel $\left(\mathrm{Na}_{\mathrm{v}} 1.2\right)$. All 3 individuals presented with seizures in early infancy. However, there were striking differences in the spectrum of clinical presentations, ranging from BFNIS to OS. A change of ion selectivity of $\mathrm{Na}_{\mathrm{v}} 1.2$ is considered to be the potential pathomechanism underlying this $\mathrm{Na}_{\mathrm{v}} 1.2$ channel dysfunction. The observation of benign and severe pheno-
\end{abstract}

\section{KARGER}

E-Mail karger@karger.com

www.karger.com/msy types due to an identical mutation within one family contradicts the hypothesis of different modes of inheritance as a mandatory feature discriminating BFNIS from SCN2A encephalopathy.

(C) 2016 S. Karger AG, Basel

Voltage-gated sodium channels $\left(\mathrm{Na}_{\mathrm{v}}\right)$ consist of a large pore-forming alpha subunit and smaller 1 or 2 auxiliary beta-subunits. They control sodium influx in response to changes of the membrane potential, therefore initiating and propagating action potentials in excitable cells along nerves, muscle fibres and neuronal somatodendritic compartments. Channel properties rely on the pore-forming alpha subunit and 9 different alpha subunits $\left(\mathrm{Na}_{\mathrm{v}} 1.1-1.9\right)$ are found in mammals. $\mathrm{Na}_{\mathrm{v}}$ channels are part of a superfamily of cation channels with 4 domains, each containing

J.R.L. and A.M. jointly supervised this work. (c) 2016 S. Karger AG, Basel

$1661-8769 / 16 / 0074-0182 \$ 39.50 / 0$
Steffen Syrbe, MD

Department of General Pediatrics, Center for Pediatrics and Adolescent Medicine University Hospital Heidelberg, Im Neuenheimer Feld 400 DE-69120 Heidelberg (Germany)

E-Mail steffen.syrbe@ med.uni-heidelberg.de 


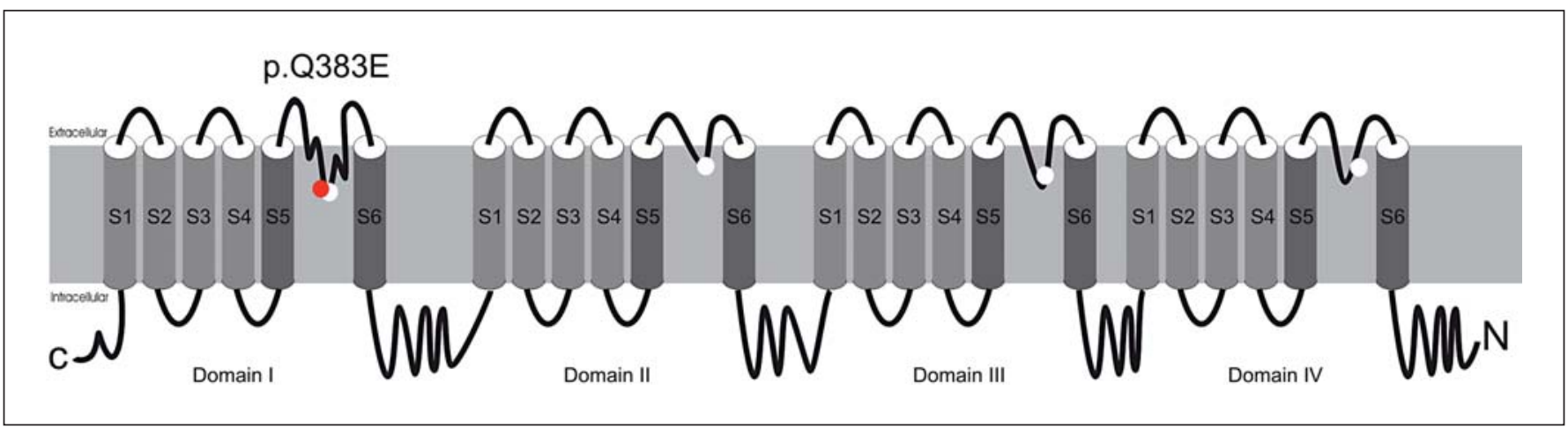

Fig. 1. Structure of the alpha subunit of $\mathrm{Na}_{\mathrm{v}}$ channels. Each alpha subunit folds into 4 domains, each containing 6 transmembrane segments (S1-6). S1-4 segments form the voltage sensing module, and S5-6 with the re-entrant P-loop serve as the pore-forming module. White circles locate the inner 'DEKA' ring of amino resi- dues from the 4 domains (D1p50, E2p50, K3p50, and A4p50) that form the narrowest part of the ion selectivity filter among eukaryotic $\mathrm{Na}_{\mathrm{v}} 1$ channels. The mutation p.Q383E (red) is adjacent to the 'DEKA' ring, resulting in an ED-motif (E1p49 and D1p50) in the P-loop of the first domain within the selectivity filter.
6 transmembrane segments (S1-6) (fig. 1). The selectivity for the different cations $\left(\mathrm{Na}^{+}, \mathrm{Ca}^{2+}\right)$ is determined by the selectivity filter within the membrane re-entrant loop ( $\mathrm{P}$ loop) between the pore-forming S5 and S6 segments of each domain [Mantegazza and Catterall, 2012; Stephens et al., 2015]. SCN2A encodes the pore-forming sodium channel protein type 2 alpha subunit $\left(\mathrm{Na}_{\mathrm{v}} 1.2\right)$, the major alpha subunit in excitatory neurons [Mantegazza and Catterall, 2012; Howell et al., 2015]. Familial mutations in $S C N 2 A$ have been identified as a cause of benign familial neonatal-infantile epilepsy (BFNIS; OMIM 607745) with self-limited seizures and normal intellectual development [Heron et al., 2002; Herlenius et al., 2007]. Recently, more severe phenotypes, such as Ohtahara syndrome (OS), West syndrome, and epilepsy of infancy with migrating focal seizures (EIMFS) have been associated with de novo mutations in SCN2A (early infantile epileptic encephalopathy, type 11, OMIM 613721) [Ogiwara et al., 2009; Nakamura et al., 2013; Howell et al., 2015].

We describe a family that harbours a prominently located novel missense variant in $S C N 2 A$, predisposing to a wide range of epileptic disorders with onset in infancy.

\section{Materials and Methods}

In a girl with OS, we performed targeted panel sequencing of epilepsy genes as previously described [Lemke et al., 2012]. We reviewed the clinical and genetic information on this patient and its relatives. Segregation analysis of a suspicious sequence alteration was performed using conventional Sanger sequencing. Predictions of the functional impact was assessed by different in silico analysis tools (PolyPhen2, http://www.genetics.bwh.harvard.edu/ pph2 and MutationTaster, http://www.mutationtaster.org) using the SCN2A transcript number ENST00000375437. Variants were then compared to 60,706 controls of the ExAC browser (http:// exac.broadinstitute.org/) and in-house data. Conservation of mutated positions was evaluated using sequence alignment of different species and different $\mathrm{Na}_{\mathrm{v}}$ channels.

\section{Computer Modelling of Wild Type and Mutated $\mathrm{Na}_{v} 1.2$}

The X-ray structure of the bacterial sodium channel $\mathrm{Na}_{\mathrm{v}} \mathrm{Ab}$ [Payandeh et al., 2011] was used as a template to build a closedstate model of the $\mathrm{Na}_{\mathrm{v}} 1.2$ channel alpha subunit as described elsewhere [Du et al., 2013]. We used the adjusted sequence alignment between P2 helices of $\mathrm{Na}_{\mathrm{v}} \mathrm{Ab}$ and $\mathrm{Na}_{\mathrm{v}} 1$ channels [Tikhonov and Zhorov, 2012]. Homology modelling and ligand docking were performed using the ZMM program [Garden and Zhorov, 2010] and Monte Carlo-minimization protocol [Li and Scheraga, 1987] as described elsewhere [Du et al., 2013]. Molecular images were created using the PyMol Molecular Graphics System, Version 0.99rc6 (Schrödinger, LLC, New York, N.Y., USA).

To preclude large deviations of the channel backbones from the $\mathrm{X}$-ray templates during energy minimizations (and thus preserve the channel folding), a set of distance constraints (pins) is imposed between matching alpha carbons in the template and the model. A pin constraint is a flat-bottom parabolic energy function that allows an atom (in this study, an alpha carbon) to deviate penalty free of up to $1 \AA$ from the template and imposes a penalty of $10 \mathrm{kcal}$ $\mathrm{mol}^{-1} \AA^{-1}$ for larger deviations.

To overcome the problem of different residue numbers in homologous positions of different sodium channels and to highlight symmetric locations of residues in different channel repeats, we used a residue-labelling scheme, which is universal for P-loop channels [Zhorov and Tikhonov, 2004; Du et al., 2013]. A residue label includes the repeat number (1-4), segment type (p, P-helix), and relative number of the residue in the segment. According to this scheme, selectivity-filter residues D, E, K, and A in repeats IIV are designated, respectively, D1p50, E2p50, K3p50, and A4p50. 

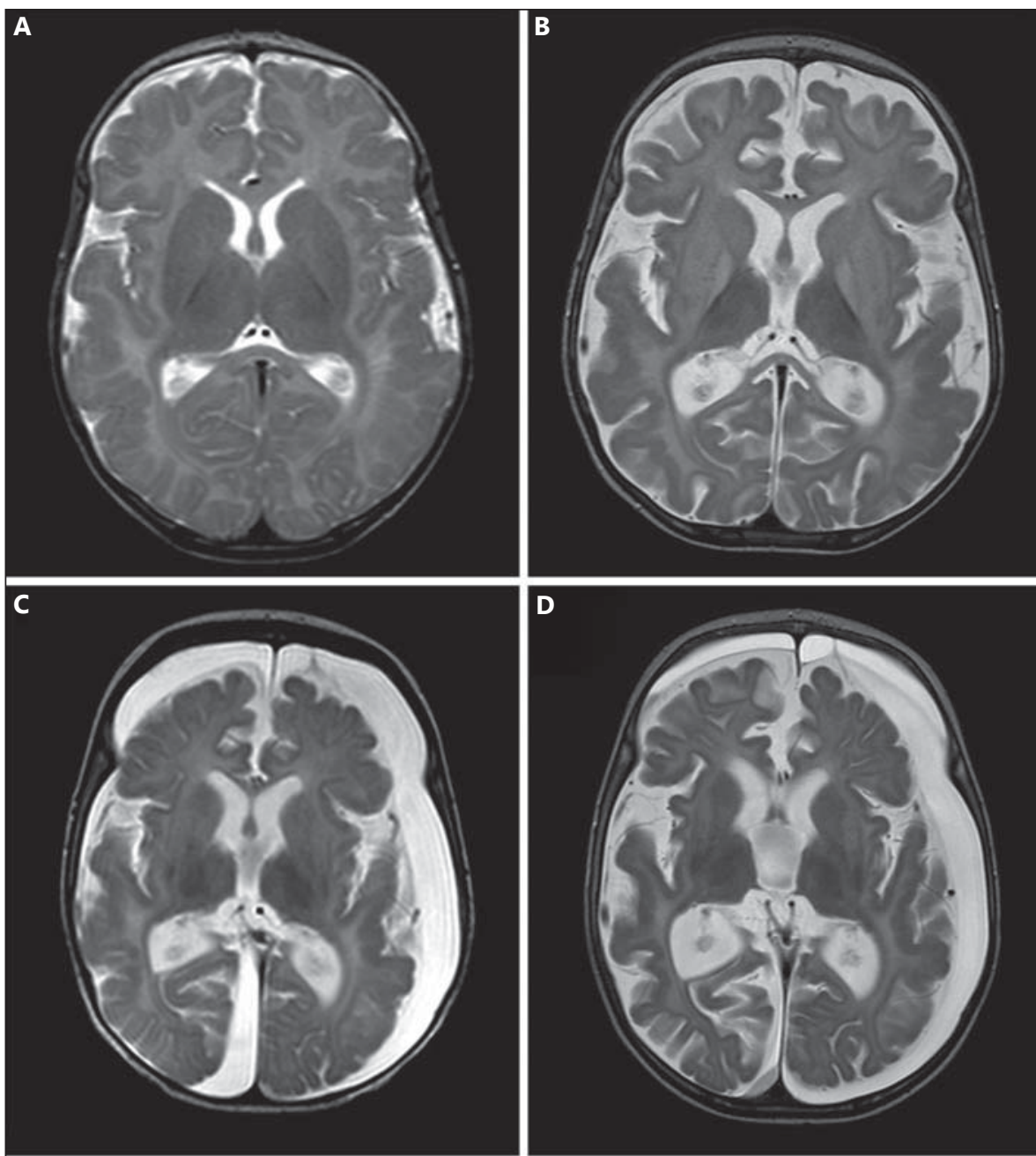

Fig. 2. Brain MRI of the IP with OS. Axial T2-weighted images at seizure onset at an age of 3 months (A) and at 4 (B), 5 (C) and at an age of 6 months (D), respectively, demonstrating progressive supratentorial brain atrophy. EEG at 14 weeks displaying burstsuppression pattern (E) and onset of a tonic seizure (F). Both are the characteristic features of OS.
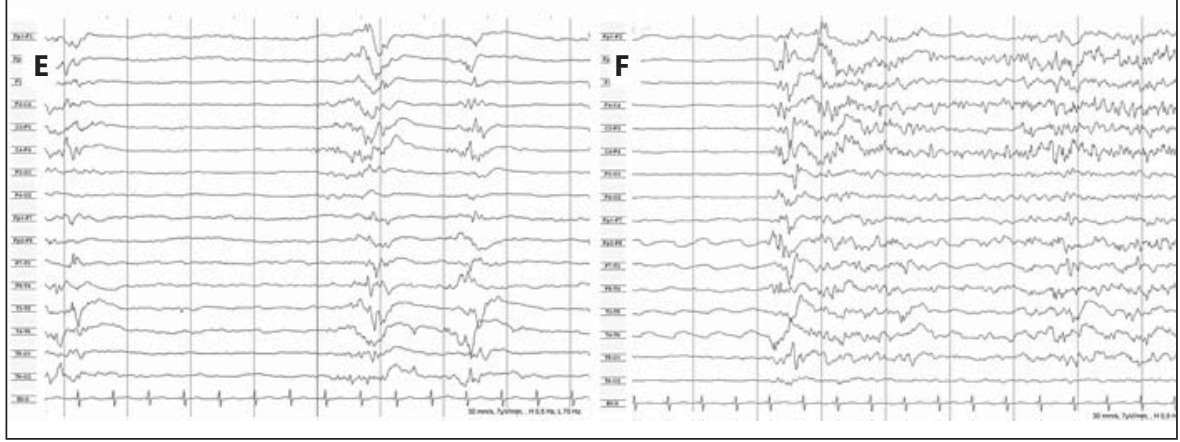

\section{Results}

\section{Clinical Features}

The index patient (IP; III.1) is a girl, born eutrophic at term. Repetitive tonic seizures with initial crying were observed up to 5 times per day from 11 weeks of age. Seizures occurred in clusters and remained despite therapy with sulthiame, valproic acid, pyridoxine, clobazam, phe- nytoin, thiopental, levetiracetam, topiramate, and oxcarbazepine (OXC). Interictal electroencephalography (EEG) was described as normal at onset when tonic seizures of different origins were recorded. Follow-up EEGs showed a slowing of background activity with increasingly interspersed focal spikes, poly-spikes and sharp waves, evolving into a burst-suppression pattern within 3 weeks from seizure onset (fig. 2). With decreasing seizure 
Fig. 3. Pedigree of the family. IP with OS (arrow) as well as her paternal half-brother (III.2) with benign infantile seizures both carry the SCN2A missense mutation p.Q383E. The mutation was excluded in both paternal grandparents (I.1 and I.2), putatively occurring de novo in the father (II.2) who showed an intermediate phenotype of SCN2A-related infantile epilepsy. ID = Intellectual disability.

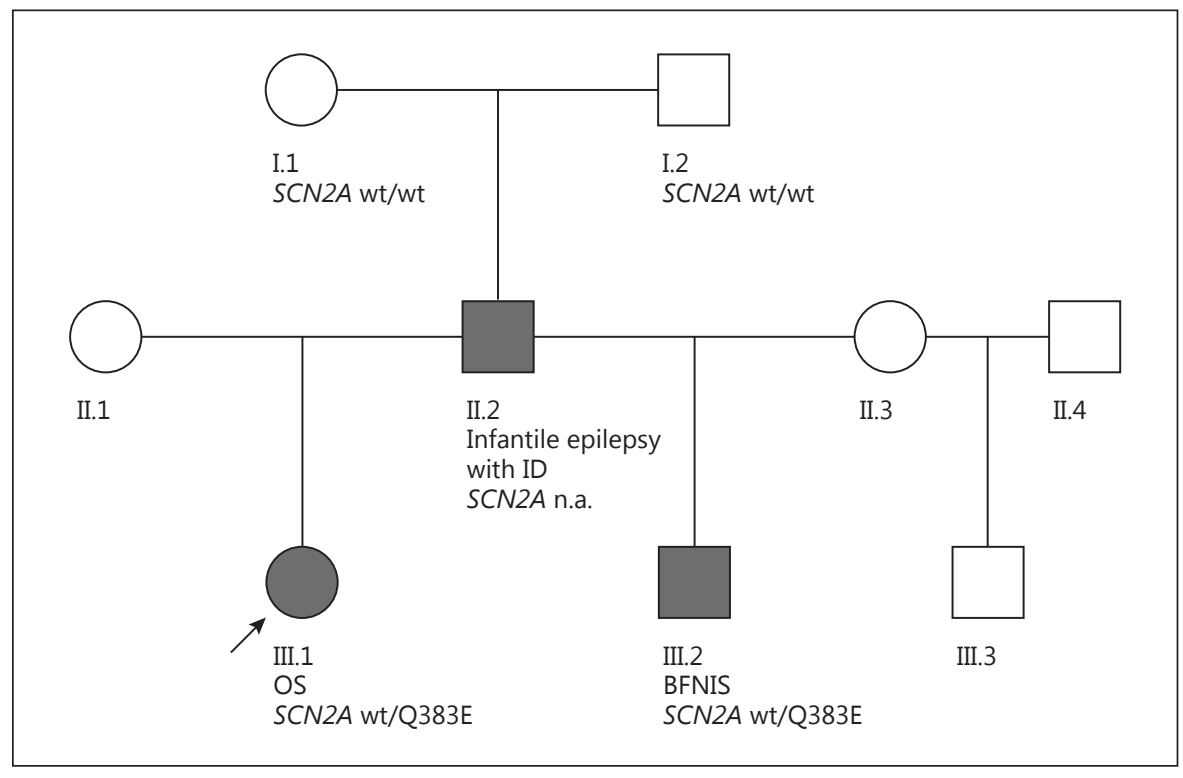

frequency after 4 months of age, the EEG evolved into generalized slow delta activity and only occasional spikes (18 weeks); after 6 months of age the EEG showed low voltage undifferentiated activity with frequent ictal pattern and occasional focal spikes and sharp waves.

Brain MRI was normal at 3 months of age, developing severe supratentorial brain atrophy up to 6 months with progressive subdural hygroma that was treated with subduroperitoneal shunting. The girl's development at 7 years is severely impaired with global developmental delay, bilateral cerebral palsy (Gross Motor Function Classification System level V) and absent social interaction and communicative skills. Diverse seizure types are frequently reported, including dyscognitive, clonic, gelastic seizures, and generalized tonic seizures.

Extensive diagnostic work up, including metabolic investigations, muscle biopsy, repeated cerebrospinal fluid analysis and genetic testing with comparative genomic hybridization and SCN1A and CDKL5 mutation screening was not able to identify an underlying cause of disease.

The father (II.2) of the IP showed frequent clusters of idiopathic focal clonic, generalized tonic and tonic-clonic seizures from his third month of life. Seizures were not controlled with phenobarbital and clonazepam and persisted through childhood into adulthood with up to 8 seizures per month. Seizures were later described as generalized tonic-clonic seizures and dyscognitive seizures. After a prolonged unilateral clonic seizure with left-sided postictal paresis at 2 years of age, he experienced an encephalopathic state with reduced consciousness lasting 6 days.

Clinical Variability Associated with a

Novel SCN2A Mutation
EEG during the first years of life remained without clear epileptic discharges but showed slowing of background activity. EEG after 6 years displayed focal spikes and sharp waves and intermittent alternating slowing. Computed tomography at 3 years of age showed no abnormalities. While motor development was normal, mild intellectual disability and behavioural abnormalities developed. At current age of 31 years, occasional seizures were reported despite medication with OXC. He declined genetic testing.

The paternal half-brother (III.2) was born eutrophic at term. Focal sleep-related seizures started 2 days after his first vaccination at 9 weeks of life. He suddenly arose from sleep with crying, scared staring eye gaze, and tonic hand posturing. Clonic and tonic-clonic seizures occurred soon after, still in the third month of life, and were partially accompanied by ictal vomiting. MRI at 3 months was normal. Levetiracetam treatment was introduced at 10 weeks of age, but clusters of seizures remained up to 4 months when OXC was added. Apart from one fever-related cluster of seizures at 5 months, he became seizure free up to 3 years when occasional dyscognitive and focal clonic seizures recurred. Neuropsychological testing at 4 years showed a minor non-significant delay with finemotor skills and language performance being normal for age.

Genetic Testing

In the IP with OS, we identified the heterozygous missense variant FOLR1 c.292C > T, p.R98W and the hetero- 


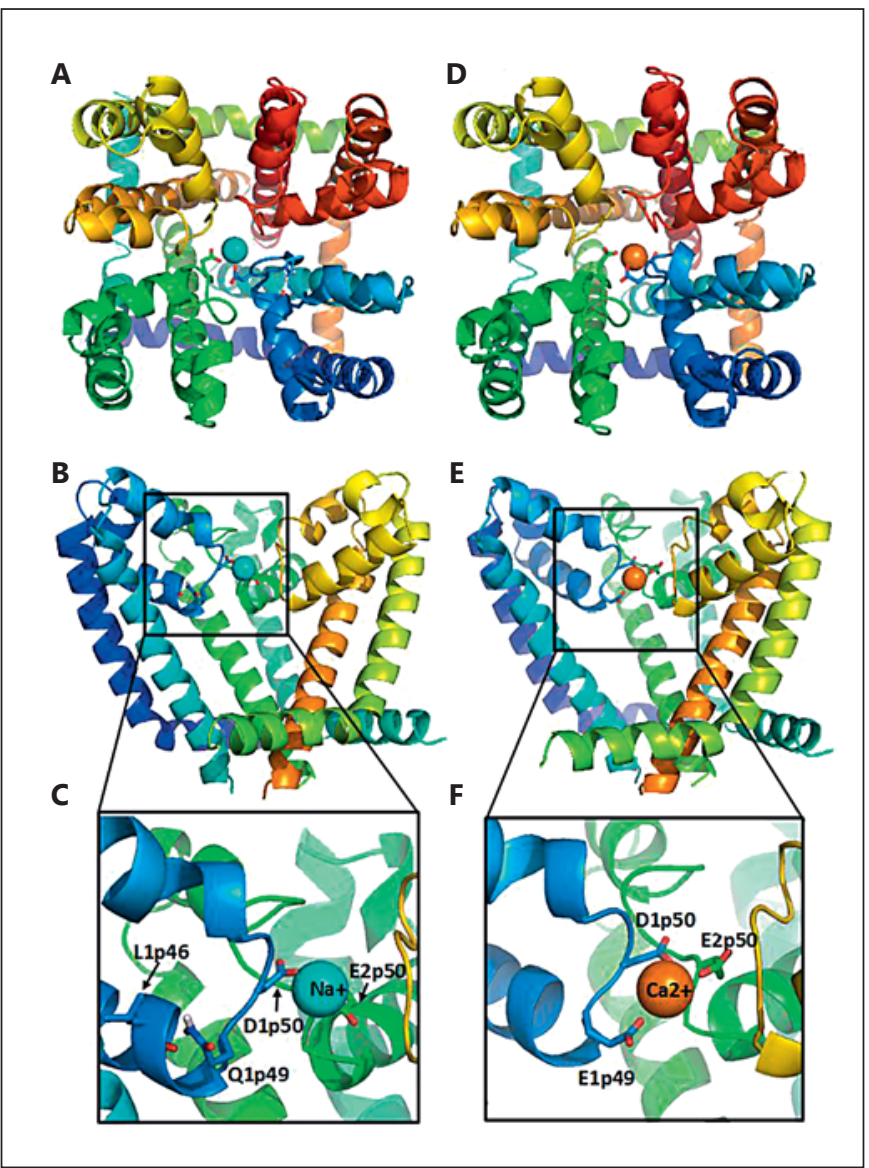

Fig. 4. $\mathrm{Na}_{\mathrm{v}} \mathrm{Ab}$-based homology model of the closed $\mathrm{Na}_{\mathrm{v}} 1.2$ channel pore domain with a sodium ion $(\mathbf{A}-\mathbf{C})$ and the $\mathrm{Na}_{\mathrm{v}} 1.2$ mutant Q1p49E with a calcium ion (D-F). Repeats I, II, III, and IV are coloured blue, green, yellow, and red. Side chains of the selectivity filter residues D1p50 and E2p50 as well as Q1p49 or E1p49 are shown as sticks. A, D Extracellular views. B, E Side views from the lipids with repeat IV removed for clarity. C, $\mathbf{F}$ Enlarged views of $\mathbf{B}$ and $\mathbf{E}$, respectively.

zygous missense variant SCN2A c.1147C >G, p.Q383E. Standard prediction tools (PolyPhen2 and MutationTaster) predicted the FOLR1 variant as being benign. According to the ExAC browser, it has been reported in 400 controls yielding a minor allele frequency of 0.003295 . In contrast, the variant $S C N 2 A$ c. $1147 \mathrm{C}>\mathrm{G}, \mathrm{p} . \mathrm{Q} 383 \mathrm{E}$ is predicted as likely pathogenic with a very high confidence score $(>0.99)$ and is absent in the ExAC browser. The variant segregated in the paternal half-brother of the IP. The father was unavailable for genetic testing but remains an obligate carrier of the $S C N 2 A$ variant. The variant was excluded in both paternal grandparents (I.1 and I.2) (fig. 3).
Glutamine at position 383 (or homologue positions in different $\mathrm{Na}_{\mathrm{v}}$, corresponding to position $1 \mathrm{p} 49$ according to the universal residue-labelling scheme for P-loop channels [Zhorov and Tikhonov, 2004; Du et al., 2013]) is phylogenetically highly conserved in all eukaryotic $\mathrm{Na}_{\mathrm{v}} 1$ channels affecting the motif of the selectivity filter in the P-loop within the pore region of $\mathrm{Na}_{\mathrm{v}} 1.2$. Glutamine Q1p49 is located in immediate proximity to residue D1p50. The latter contributes to the so-called DEKA ring $\left(\mathrm{D}^{1 \mathrm{p} 50}, \mathrm{E} 2 \mathrm{p} 50, \mathrm{~K} 3 \mathrm{p} 50\right.$, and $\left.\mathrm{A} 4 \mathrm{p} 50\right)$ that constitutes the high-field strength site of the selectivity filter in the Ploop of Nav1.2 [Heinemann et al., 1992].

\section{Computed Modelling of Mutated Selectivity Filter of}

$N a_{v} 1.2$

We Monte Carlo energy-minimized the homology model of the $\mathrm{Na}_{\mathrm{v}} 1.2$ channel and then docked a sodium ion in the selectivity filter region (fig. 4). In low-energy structures, the sodium ion was coordinated by the selectivity filter residues D1p50 and E2p50. The side chain of the lysine residue $\mathrm{K} 3 \mathrm{p} 50$ adopted various side-chain conformations (not shown) so that the selectivity filter region had an electrostatic balance of 2 positive (K3p50 and $\mathrm{Na}^{+}$) and 2 negative (D1p50 and E2p50) charges. Residue Q1p49 donated an H-bond to the backbone carbonyl of L1p46 (fig. 4C).

We then created a homology model of the Q1p49E mutant and docked a calcium ion in the selectivity filter region to ensure the electrostatic balance between 3 positive (K3p50 and $\mathrm{Ca}^{2+}$ ) and 3 negative (E1p49, D1p50, and E2p50) charges. The E1p49 side chain lacks a polar hydrogen, capable to donate an $\mathrm{H}$-bond to the backbone carbonyl of L1p46. In our model, the negatively charged group of E1p49 was attracted to the $\mathrm{Ca}^{2+}$ ion, which was coordinated by $\mathrm{D}^{1 \mathrm{p} 50}$ and $\mathrm{E}^{2 \mathrm{p} 50}$ (fig. $4 \mathrm{D}-\mathrm{F}$ ).

\section{Discussion}

We identified a novel SCN2A mutation in a family with 3 different epilepsy phenotypes starting in early infancy between 2 and 3 months of age which corresponds to the reported seizure onset in SCN2A-related epilepsies [Herlenius et al., 2007; Ogiwara et al., 2009; Nakamura et al., 2013]. The IP was diagnosed with OS, her clinical course and the severe brain atrophy (fig. 2) highly resemble the described SCN2A-related epileptic encephalopathy [Nakamura et al., 2013]. The onset of brain atrophy led to formation of subdural hygroma in this girl, which argues for a rapid loss of brain volume in this case. The 
paternal half-brother showed seizures between 2 and 5 months of life and an age-appropriate psychomotor development, which is in good agreement with BFNIS. Persistence of seizures after the first year of life is uncommon but has been observed in BFNIS [Herlenius et al., 2007]. The father of both children showed an intermediate epilepsy phenotype according to the classification of Howell et al. [2015] displaying treatment resistant partial seizures, encephalopathic episodes that started in early infancy, intellectual disability, and behavioural changes at age 31 . To the best of our knowledge, he is the oldest patient reported with this intermediate phenotype, and his clinical course suggests persistence of seizures into adulthood in this group of affected individuals. It is difficult to judge treatment response in this family, while no medication appeared to be effective in the girl with OS and her father with the intermediate phenotype; introduction of OXC coincided with seizure freedom in her paternal halfbrother. However, this may be due to the self-limiting character of the disorder rather than resulting from beneficial therapy response. Interestingly, seizure onset in her half-brother coincided with his first vaccination, and he displayed 1 fever-related cluster of seizures at 5 months, a feature well-known from SCN1A-related epilepsies [Mancardi et al., 2006; Zuberi et al., 2011].

Both children carry the novel mutation p.Q383E in the $\mathrm{Na}_{\mathrm{v}} 1.2$ channel. The father, who was not available for genetic testing, is an obligate carrier of SCN2A p.Q383E, most likely in a de novo state. In line with previous reports on SCN2A-related epilepsies, the detection of the missense variant p.Q383E suggests that haploinsufficiency is not the underlying cause. The mutation p.Q383E is predicted to change protein function and is absent in the ExAC browser. p.Q383E affects the extracellular membrane re-entrant P-loop of the domain I of $\mathrm{Na}_{\mathrm{v}} 1.2$. Glutamine at position 383 is highly conserved in eukaryotic $\mathrm{Na}_{\mathrm{v}} 1$ channels and is located in the immediate proximity to the selectivity filter, determining sodium specificity (fig. 1). The neighbouring aspartate at position 384 is a part of the high-field strength site of the selectivity filter, the so-called 'DEKA (Asp-Glu-Lys-Ala) ring' (equalling $\mathrm{D} 1 \mathrm{p} 50, \mathrm{E} 2 \mathrm{p} 50, \mathrm{~K} 3 \mathrm{p} 50$, and $\mathrm{A} 4 \mathrm{p} 50$ ). The ring is formed by residues between P-loop helices from all 4 channel domains (I-IV). Residues at these positions are evolutionary conserved in all voltage-gated cation channels and determine the $\mathrm{K}^{+}, \mathrm{Ca}^{2+}, \mathrm{Na}^{+}$, or mixed ion selectivity [Stephens et al., 2015].

The homologue P-loop residue in the widely studied $\mathrm{Na}_{\mathrm{v}} 1.1$ channel (SCN1A p.Q381) has been spared from mutations and polymorphism so far, and reported mis-

Clinical Variability Associated with a

Novel SCN2A Mutation sense mutations affecting neighbouring residues of the selectivity filter of $\mathrm{Na}_{\mathrm{v}} 1$ [e.g., de novo SCN1A p.F383D, which corresponds to residue F385 (equalling F1p51) in $\mathrm{Na}_{\mathrm{v}} 1.2$ ] resulted in Dravet syndrome, supporting the vital role of this pore region [Mancardi et al., 2006; Zuberi et al., 2011] (ExAc browser; SCN1A variant database, http:// www.molgen.vib-ua.be/SCN1AMutations/). A gain of function of $\mathrm{Na}_{\mathrm{v}} 1.2$ has been proposed to underlie hyperexcitability in some epilepsy-associated SCN2A mutations; however, conflicting results of in vitro electrophysiological analyses apply for a more complex dysfunction [Ogiwara et al., 2009]. The mutation p.Q383E results in a conformational change within the selectivity filter with a newly formed ED-motif in the first domain of $\mathrm{Na}_{\mathrm{v}} 1.2$, showing resemblance to the selectivity filter residues in the second domain of calcium ion channels [Stephens et al., 2015]. Therefore, we hypothesized that a change from sodium selectivity to calcium selectivity can underlie the channel dysfunction and created a computer model of the mutant channel (fig. 4). While this model supports a possible change of ion selectivity from the wild-type channel to the $\mathrm{Q}^{1 \mathrm{p} 49} \mathrm{E}$ (p.Q383E) mutant, it is not precise enough to predict if calcium ions will be permeating through the channel or block the channel. Electrophysiological studies of the mutant channel are necessary to test this hypothesis.

Despite no clear genotype-phenotype correlation, different modes of inheritance are assumed for BFNIS and SCN2A-related epileptic encephalopathy [Howell et al., 2015]. In a minority of patients with Dravet syndrome, relatives have been reported with the more benign generalized epilepsy with febrile seizures plus (GEFS+) carrying the same SCN1A mutation [Goldberg-Stern et al., 2014; Scheffer, 2011]. Recently, a similar phenotypic variability within one family has been documented for KCNT1 ranging from benign autosomal dominant nocturnal frontal lobe epilepsy to EIMFS [Møller et al., 2015]. Our report adds $S C N 2 A$ as another gene with highly variable penetrance of specific mutations, demonstrating that a de novo mutation, which is associated initially with an intermediate epileptic phenotype, can result in benign or more severe epileptic syndromes in the second generation. SCN9A has been proposed as one possible modifier of disease in Dravet syndrome [Singh et al., 2009]. We were not able to identify additional variants in $S C N 9 A$ or other known epilepsy genes tested in our IP with OS; however, we cannot rule out modifying variants in other 'non-epilepsy' genes, affecting the course of epilepsy through changes within other metabolic or signaling pathways. Our observation illustrates the complexity of the growing 
field of recognized channelopathies in genetic epilepsies and the difficulties of assessing the pathogenicity of a certain variant, which renders genetic counselling of affected families challenging. Other, yet unknown, modifying factors likely contribute to the severity of disease.

\section{Acknowledgement}

We are grateful to all patients and their families for their participation in this study.

\section{Statement of Ethics}

The study was approved by local authorities (University of Leipzig).

\section{Disclosure Statement}

The authors declare no conflicts of interest.

\section{References}

Du Y, Nomura Y, Satar G, Hu Z, Nauen R, et al: Molecular evidence for dual pyrethroid-receptor sites on a mosquito sodium channel. Proc Natl Acad Sci USA 110:11785-11790 (2013).

Garden DP, Zhorov BS: Docking flexible ligands in proteins with a solvent exposure- and distance-dependent dielectric function. J Comput Aided Mol Des 24:91-105 (2010).

-Goldberg-Stern H, Aharoni S, Afawi Z, Bennett O, Appenzeller S, et al: Broad phenotypic heterogeneity due to a novel SCN1A mutation in a family with genetic epilepsy with febrile seizures plus. J Child Neurol 29:221-226 (2014).

-Heinemann SH, Terlau H, Stühmer W, Imoto K, Numa S: Calcium channel characteristics conferred on the sodium channel by single mutations. Nature 356:441-443 (1992).

- Herlenius E, Heron SE, Grinton BE, Keay D, Scheffer IE, et al: SCN2A mutations and benign familial neonatal-infantile seizures: the phenotypic spectrum. Epilepsia 48:11381142 (2007).

Heron SE, Crossland KM, Andermann E, Phillips HA, Hall AJ, et al: Sodium-channel defects in benign familial neonatal-infantile seizures. Lancet 360:851-852 (2002).

Howell KB, McMahon JM, Carvill GL, Tambunan D, Mackay MT, et al: SCN2A encephalopathy: a major cause of epilepsy of infancy with migrating focal seizures. Neurology 85 : 958-966 (2015).
Lemke JR, Riesch E, Scheurenbrand T, Schubach M, Wilhelm C, et al: Targeted next generation sequencing as a diagnostic tool in epileptic disorders. Epilepsia 53:1387-1398 (2012).

Li Z, Scheraga HA: Monte Carlo-minimization approach to the multiple-minima problem in protein folding. Proc Natl Acad Sci USA 84 6611-6615 (1987).

- Mancardi MM, Striano P, Gennaro E, Madia F, Paravidino R, et al: Familial occurrence of febrile seizures and epilepsy in severe myoclonic epilepsy of infancy (SMEI) patients with SCN1A mutations. Epilepsia 47:1629-1635 (2006).

Mantegazza M, Catterall WA: Voltage-gated Na+ channels: structure, function, and pathophysiology, in Noebels JL, Avoli M, Rogawski MA, et al (eds): Jasper's Basic Mechanisms of the Epilepsies, ed 4 (Oxford University Press, New York 2012).

Møller RS, Heron SE, Larsen LH, Lim CX, Ricos MG, et al: Mutations in KCNT1 cause a spectrum of focal epilepsies. Epilepsia 56:e114120 (2015).

Nakamura K, Kato M, Osaka H, Yamashita S, Nakagawa E, et al: Clinical spectrum of $S C N 2 A$ mutations expanding to Ohtahara syndrome. Neurology 81:992-998 (2013).

Ogiwara I, Ito K, Sawaishi Y, Osaka H, Mazaki E, et al: De novo mutations of voltage-gated sodium channel alpha II gene SCN2A in intractable epilepsies. Neurology 73:1046-1053 (2009).
Payandeh J, Scheuer T, Zheng N, Catterall WA: The crystal structure of a voltage-gated sodium channel. Nature 475:353-358 (2011).

Scheffer IE: Does genotype determine phenotype? Sodium channel mutations in Dravet syndrome and GEFS+. Neurology 76:588 (2011).

- Stephens RF, Guan W, Zhorov BS, Spafford JD: Selectivity filters and cysteine-rich extracellular loops in voltage-gated sodium, calcium, and NALCN channels. Front Physiol 6:153 (2015).

-Singh NA, Pappas C, Dahle EJ, Claes LR, Pruess $\mathrm{TH}$, et al: A role of SCN9A in human epilepsies, as a cause of febrile seizures and as a potential modifier of Dravet syndrome. PLoS Genet 5:e1000649 (2009).

Tikhonov DB, Zhorov BS: Architecture and pore block of eukaryotic voltage-gated sodium channels in view of NavAb bacterial sodium channel structure. Mol Pharmacol 82:97-104 (2012).

Zhorov BS, Tikhonov DB: Potassium, sodium, calcium and glutamate-gated channels: pore architecture and ligand action. J Neurochem 88:782-799 (2004).

Zuberi SM, Brunklaus A, Birch R, Reavey E, Duncan J, Forbes GH: Genotype-phenotype associations in SCN1A-related epilepsies. Neurology 76:594-600 (2011). 